\title{
Development of medical records exchange system - a case of OpenMRS and Care2X
}

\author{
Geofrey Rweikiza $^{1 *}$ and Dina Machuve ${ }^{2}$ \\ ICT officer at the Tanzania Communications Regulatory Authority (TCRA), Tanzania ${ }^{1}$ \\ Lecturer, Nelson Mandela African Institution of Science and Technology (NM-AIST) in Tanzania ${ }^{2}$
}

Received: 01-January-2019; Revised: 24-March-2019; Accepted: 27-March-2019

(C)2019 Geofrey Rweikiza and Dina Machuve. This is an open access article distributed under the Creative Commons Attribution (CC BY) License, which permits unrestricted use, distribution, and reproduction in any medium, provided the original work is properly cited.

\begin{abstract}
A significant number of healthcare delivery services in developing countries continue to adopt eHealth systems, enabled by information and communication technology (ICT). Open source health information systems (HIS) such as OpenMRS and Care $2 X$ are increasingly adopted in resource constrained countries including Tanzania. The exchange of patients' information in terms of electronic medical records (EMR) between different types of HIS is challenged by the heterogeneity. This brings challenges in integrating medical data and also interoperability of the e-medical recording systems became complicated. In this study, we proposed a medical records exchange system (MRES) prototype for exchange of information on OpenMRS and Care2x hospital information systems. The software development was done using Agile methodology and implemented in a web-based application framework that allowed for ease of integration and use of health information systems at medical facilities. The MRES allows for exchange of medical information when patients are transferred from one hospital to another overcoming the queues and paper work involved in the process.
\end{abstract}

\section{Keywords}

E-medical recording system, Medical records, Data integration.

\section{Introduction}

Information and communication technologies (ICTs) have a significant role in transforming the lives of Tanzanians and individuals across the world in different aspects of education, health, and governance. It is acknowledged that ICT has the potential to transform the support and delivery of healthcare services in both developed and developing countries.

Electronic health (e-health) refers to the use of ICT for cost-effective and secure delivery of health care services [1]. The range of services includes electronic health records, telehealth, mobile health services (mHealth), and decision support systems for health [2]. The health care delivery services are challenged with systemic misalignment of incentives, health records, lack of coordination, that spawns' inefficient allocation of resources or harm to patients collectively termed fragmentation [3].

\footnotetext{
*Author for correspondence
}

Electronic medical records are generally fragmented across multiple treatment sites which means patients' e-medical records are distributed in different health care services which have different types of e-medical recording systems.

This has brought challenges in sharing information between e-medical recording systems. Fragmentation occurs when there is heterogeneity among the systems which means that e-medical recording systems have different characteristics and behaviours but they all perform similar functions.

An electronic medical record system (or electronic health record system) is an electronic record of health-related information on an individual that can be created, gathered, managed, and consulted by authorized clinicians and staff within one health care organization, have the potential to provide substantial benefits to physicians, clinical practices, and health care organizations [4]. These systems can facilitate workflow and improve the quality of patient care and patient safety [4, 5]. However, structural barrier on the data model is one of the factors contributing to 
challenges in sharing of information across e-medical records systems [6]. The inconsistency on data models arise from the use of multiple platforms from multiple vendors in e-medical recording systems. This causes a significant challenge on electronic health records, adoption in terms of connectivity across health facilities and respective stakeholders (e.g. physicians) [7]. The lack of data standards and complex electronic medical recording systems is a known major barrier in the exchange process of patients' information $[8,9]$.

This study, therefore, seeks to reduce fragmentations among e-medical recording system data models by developing integrated data models of e-medical records. Data Model is one among the major barrier to effective sharing of information in an e-medical recording system. It is therefore important to develop a mechanism or engine which can be used to integrate the systems so that they can be able to share information.

\section{Health information systems for hospitals in Tanzania}

Health services in Tanzania are provided by various stakeholders with major providers being the government, parastatal organizations, religious organizations, private practitioners, traditional medicine and voluntary organizations [10]. The delivery of health services in Tanzania is pyramidal in structure [11]. The bottom and the first level are village health services, followed by dispensary health services, health center services, district hospitals, regional hospitals and referral hospitals at the top [12, 13].

Statistics indicate that in Tanzania, 55 hospitals operate at district level out of which 13 are owned by faith-based organizations (FBO). There are 18 hospitals operating at regional level and 8 consultancy hospitals [14]. Health centers in villages operate at the lowest level of health care delivery. They provide preventive health services that normally can be offered on homes. Dispensaries on the next level provide a range of preventive and curative measures for antenatal care, family planning, delivery and postnatal care. In addition to services offered by dispensaries, health centers provide basic laboratory services [15]. Every district in the country is supposed to have a district hospital by legislation. Similarly, in every region, a regional hospital. Regional Hospitals offer similar services to those at the district level; however, they have specialists in various health fields and offer additional services which are not provided at district hospitals.

Referral hospitals are on the top most level in Tanzania. These include Kilimanjaro Christian Medical Centre (KCMC), Mbeya Hospital, Bugando Hospital and Muhimbili National Hospital (MNH) [16]. The referral hospitals provide specialized care to patients referred from lower level hospitals [17]. In developing countries, including Tanzania, there is no clear definition of the types of services to be provided by each level of the health services in the pyramidal structure. The aim of the health care pyramidal structure in Tanzania is enabling patients to acquire health services starting from a lower level hospital to a higher level. In reality, many people directly start at the referral hospitals without even going through the lower level hospitals [14]. The extensive paper-based operations at referral hospitals in turn affect services at lower levels. Extensive paper works involved during the process of referring patients to higher level hospitals lead to health care providers at the lower level hospitals in doubt to actually refer patients [18]. Health information is isolated within hospitals limiting accessibility of the information when there is a need for a person or another health center to access the information [19]. When the specialty in the medical field (surgeon, specialists in pediatrics, cancer to mention a few) is required, the transfer of responsibility for patient care between hospitals becomes unavoidable. This in turn creates an urgent need to improve on the communication and information transfer between inpatient and outpatient physicians at the hospital [20]. The existing health information systems in hospitals in Tanzania are summarized herewith below:

\subsection{Care 2X System}

Care $2 \mathrm{x}$ is an open source web based integrated healthcare information system. The system has been operational since 2014 and the source code is open for customization by the client. The system functions on both Windows and Linux operating systems (www.care2x.org). Several health care centers and major hospitals in Tanzania, currently use Care $2 \mathrm{X}$ health information systems. These include Selian hospital, St. Elizabeth Hospital, Arusha Lutheran Medical Hospital (ALMC) in Arusha, Kilimanjaro Christian Medical Centre (KCMC) and Hydom Lutheran hospital in Mbulu [21].

Care 2x hospital information system (HIS) uses opensource software. The software includes the Apache web server and MySQL relational database 
management system. The Care2x HIS is rich in clinical features for handling several hospital operations [22]. Care2x is built using PHP (Hypertext Preprocessor). It can be integrated also with WebERP for accounts activities like issuing of invoices and its data model works only with MySQL relational database management systems rather that the SQL SERVER, DB2, PostgreSQL. Due to this fact, it has been challenging in sharing of data because of different types of data formats and structures across database management systems. Other countries that use Care2x systems include Malaysia, India, France, Italy, South Africa, Kenya and Egypt [22].

\subsection{Electronic health management system}

Electronic health management system (EHMS) is a proprietary hospital information system developed by GPITG company. The technical support for the system is provided by the same company. The system has been in use at Sanitas Hospital and Kairuki Hospital in Tanzania since 2012 (http://gpitg.com/node/38).

\subsection{Jeeva system}

Jeeva is another proprietary health information system, developed by Napier Healthcare Systems in India (http://www.napierhealthcare.com). The system has been in use at Muhimbili National Hospital (MNH) in Tanzania since 2005.

\subsection{MediPro system}

MediPro is a commercial hospital information system from by Maxcom Africa Limited (http://maxcomafrica.com). The system is in use at the Muhimbili Orthopaedic Institute (MOI) in Tanzania. MediPro is a proprietary system and the technical support is provided by the supplier.

\subsection{Government of Tanzania health management information system}

The Government of Tanzania Hospital Management Information System (GoT-HoMIS) is a full hospital management information system developed locally by the Government of Tanzania. The system is currently in use at Tumbi regional referral hospital and extended to district hospitals countrywide. GoTHoMIS operations are managed by the President's Office, Regional Administration and Local Government [23].

\subsection{District health information system}

District health information system (DHIS) is a national data warehouse for collection, validation, analysis, and presentation of aggregate statistical data, tailored to integrate health information management activities. The DHIS is an open source and open meta-data model tool with a flexible user interface which allows the user to design the contents of a specific information system with minimum knowledge of programming. DHIS2 and upwards is a modular web-based software package built with free and open source Java frameworks [24]. It runs on any platform with a Java Runtime Environment (JRE 6 or higher) installed. In Tanzania, DHIS2 is managed by the Ministry of Heath, Community Development, Gender, Elderly and Children [25]. DHIS2 software is used in more than 40 countries in Africa, Asia, and Latin America, and countries that have adopted and implemented DHIS2 as their nationwide HIS (Health Information System) software include Kenya, Tanzania (https://dhis.moh.go.tz/), Bangladesh Uganda, Liberia, Ghana, and Rwanda. An increasing number of organizations across countries are starting up new deployments of DHIS.

\subsection{0penMRS}

OpenMRS is an open source hospital information system. It is user friendly, requiring minimum programming skills to customize medical records modules [11]. It, however, requires domain expertise and knowledge in medicine. The table structure of OpenMRS system is conceptually and independent of the collected medical information or the forms to be used. This factor has the advantage to allow for customization on the basis of different user needs. A module called concept dictionary on OpenMRS stores all the diagnosis, tests, procedures, drugs, and other general questions and potential answers. The operation is achieved on the basis of the principle that information should be stored and accessible to summarize and analyze. The conceptual dictionary module allows for minimum usage of free text and maximum utilization of coded information. The components of OpenMRS are open source with free downloadable resources. The plug-in is the only component licensed in Microsoft's InfoPath program for data entry. OpenMRS is a low-cost solution for hospital information systems currently utilized in developing countries of Sub-Saharan Africa in Uganda, Kenya, Zimbabwe, Rwanda, Malawi, Tanzania, Lesotho and South America in Peru and Haiti. In Tanzania, OpenMRS is implemented at the Tanzania Training Center for International Health in Ifakara, Morogoro and Simiyu Region [11].

\section{Materials and methods}

In this study, the Agile methodology was used in software development. The Agile methodology has 
three objectives; a focus on adaptive rather than predictive methodologies; a focus on people rather than roles and thirdly a self-adaptive process [26]. The Agile development process is suitable in this research since it is difficult to predict in advance that which software requirements will persist and which will change. The Agile development consists of the following steps which are high level analysis, high level planning and a series of iterations which each iteration includes development and testing. In this methodology, it means that each module/functionality in the system undergoes a series of iterations and development on each iteration took between one and four weeks (1 to 4 weeks) to complete. It is equally a challenge to predict how customer properties will change as the research proceeds. Extreme Programming (XP) which is the most widely used Agile software development approach will establish the foundation for the development of the model. XP uses and objectoriented approach for development with four main activities: planning, design, coding and testing [26, 27].

\section{Results for medical records exchange system (MRES) prototype}

The medical records exchange system (MRES) is a prototype developed in this study for integrating data models for OpenMRS and Care $2 \mathrm{X}$ systems. It is a web-based application with a central database containing all treatment and medical history of the patients in one practice. In designing an engine, the data flow across the two systems was considered. The
MRES system has a central database which provides an exchange of data across OpenMRS and Care $2 x$ systems and it can be connected and shared / exchange data within the network and the network can be a local area network (LAN), metropolitan area network (MAN) or wide area network (WAN) or Internet. The conceptual framework of the proposed prototype is indicated in Figure $l$ and the corresponding Entity Relationship (E-R) diagram which describes the database is indicated in Figure 2.

The implementation of MRES was done in PHP and MySQL, a relational database management system on the backend. PHP is cross-platform; highly supported; open source with ease of integration on web application frameworks. On the other hand, the MySQL database was selected in the development of MRES since it is scalable and robust; open source with data integrity assurance of compliance to ACID properties.

The second iteration involved the design of "sendmail" module. In MRES the "sendmail" module is used to send email to the other hospital to provide notifications for the patients transfer to the other facility. It also contains a capability to attach any document which contains details of the patient. The development of this module involved simple mail transfer protocol (SMTP) and post office protocol version 3 (POP3). Figure 3 and 4 shows a screenshot of the send mail module on MRES.

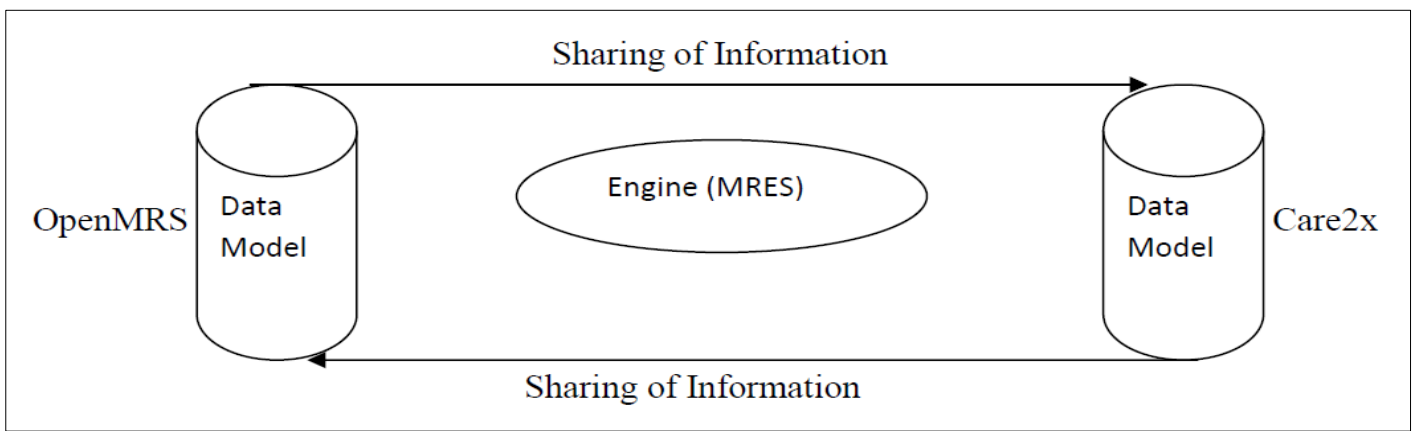

Figure 1 Conceptual framework 


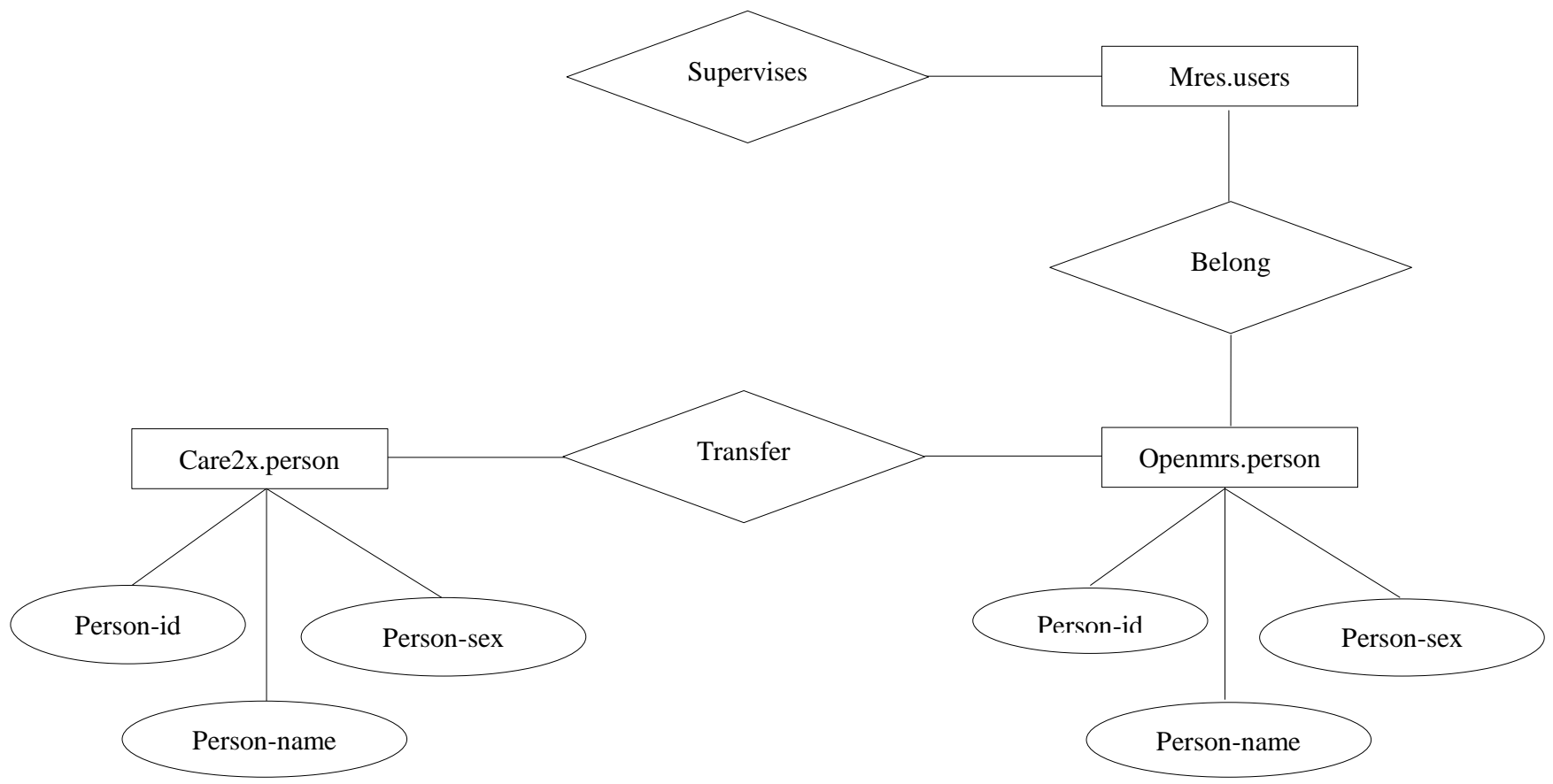

Figure 2 E-R Diagram for MRES

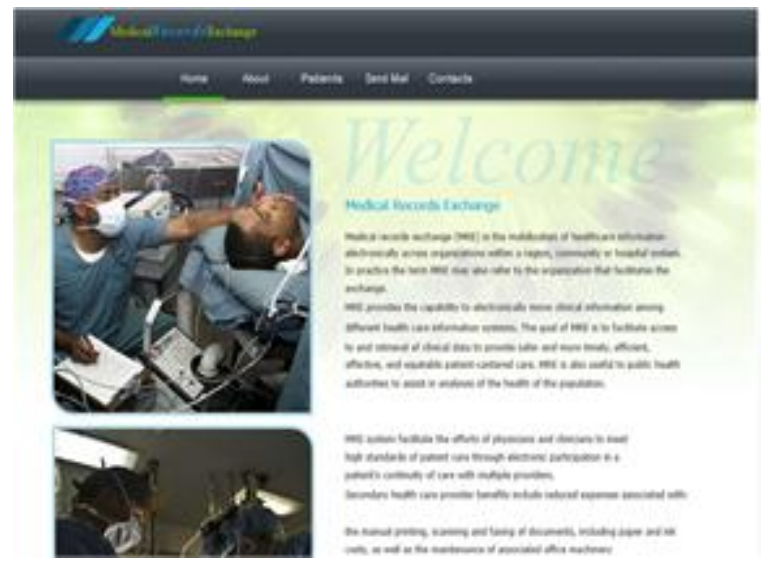

Figure 3 Homepage for the MRES

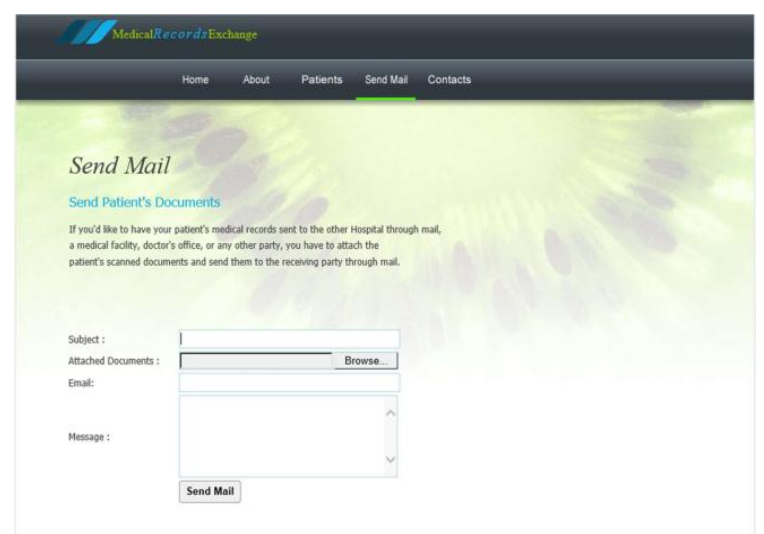

Figure 4 MRES "sendmail” module

\section{Conclusion}

MRES is one of the solutions which help to reduce fragmentation and overcoming barriers in sharing of data and exchanging information in electronic medical recording systems. The existing electronic medical recording systems have different data structure and they run on different platforms such as Windows, Linux and MAC Operating systems. They use different relational database management system like SQL server, Oracle, PostgreSQL, and MySQL. So exchange and sharing of data becomes difficult. It is recommended for future works to focus on integrating more than two electronic medical recording systems that are open-source with other commercial medical recording systems. A mobile based application for MRES is recommended to ensure patients are cared throughout. It is recommended testing of the prototype in the live environment and also further research on security issues. MRES promises to increase efficiency for the physicians and other medical staff by automating one of the most mandate and time-consuming processes. The ability to process a request in a short time period will significantly reduce costs by reducing the time spent on collecting the required information manually; improving response time for patients seeking insurance and allowing better utilization of staff resources within the practice. The MRES will allow a clinician to track data over time, easily identify which patients are due for preventative 
screenings, check how patients are doing on certain parameters such as blood pressure readings or vaccines and monitor and improve overall quality of care within the practice. An MRES will make the process of sharing of patients' information easier, more accurate and comprehensive and more efficient.

When a patient moves from one health center to the other he / she needs to start the registration process again because there is no integration between the health information systems across hospitals which will enable them to share data / information and reduce fragmentation of data. The MRES prototype provides a solution for interoperability between OpenMRS and Care2x system which by using MRES, the OpenMRS and Care $2 x$ systems can be able to share basic information of data.

\section{Acknowledgment}

The authors acknowledge the Nelson Mandela African Institution of Science and Technology (NM-AIST) through the School of Computational and Communication Science and Engineering for funding this research.

\section{Conflicts of interest}

The authors have no conflicts of interest to declare.

\section{References}

[1] https://www.who.int/goe/policies/countries/tza_ehealt h.pdf. Accessed 15 December 2018.

[2] https://www.who.int/ehealth/en/. Accessed 15 December 2018.

[3] Enthoven AC. Integrated delivery systems: the cure for fragmentation. American Journal of Managed Care. 2009; 15(10):S284-90.

[4] Kalogriopoulos NA, Baran J, Nimunkar AJ, Webster JG. Electronic medical record systems for developing countries. In annual international conference of the engineering in medicine and biology society 2009 (pp. 1730-3). IEEE.

[5] Bouidi Y, Idrissi MA, Rais N. Adopting an open source hospital information system to manage healthcare institutions. LIFE: International Journal of Health and Life-Sciences. 2017; 3(3):38-57.

[6] Omary Z, Lupiana D, Mtenzi F, Wu B. Analysis of the challenges affecting e-healthcare adoption in developing countries: a case of Tanzania. International Journal of Information Studies. 2010; 2(1).

[7] Goldstein BA, Navar AM, Pencina MJ, Ioannidis J. Opportunities and challenges in developing risk prediction models with electronic health records data: a systematic review. Journal of the American Medical Informatics Association. 2017; 24(1):198-208.

[8] $\mathrm{Wu} \mathrm{H}$, LaRue EM. Linking the health data system in the US: challenges to the benefits. International Journal of Nursing Sciences. 2017; 4(4):410-7.

[9] Tai-Seale M, Olson CW, Li J, Chan AS, Morikawa C, Durbin M, et al. Electronic health record logs indicate that physicians split time evenly between seeing patients and desktop medicine. Health Affairs. 2017; 36(4):655-62.

[10] http://moh.go.tz/en/health-services-in-tanzania. Accessed 15 December 2018.

[11] https://openmrs.org/. Accessed 28 December 2018.

[12] Winani S, Wood S, Coffey P, Chirwa T, Mosha F, Changalucha J. Use of a clean delivery kit and factors associated with cord infection and puerperal sepsis in Mwanza, Tanzania. Journal of Midwifery \& Women's Health. 2007; 52(1):37-43.

[13] Mshana S. Health management information system evaluation: lesson from Tanzania. Kuopion Yliopisto; 2004.

[14] https://extranet.who.int/nutrition/gina/sites/default/file s/TZA\%202009\%20Health\%20Sector\%20Strategic\% 20Plan\%20III.pdf. Accessed 15 December 2018.

[15] Hanson C, Ronsmans C, Penfold S, Maokola W, Manzi F, Jaribu J, et al. Health system support for childbirth care in Southern Tanzania: results from a health facility census. BMC Research Notes. 2013; $6: 1-11$.

[16] Sawe HR, Mfinanga JA, Lidenge SJ, Mpondo BC, Msangi S, Lugazia E, et al. Disease patterns and clinical outcomes of patients admitted in intensive care units of tertiary referral hospitals of Tanzania. BMC International Health and Human Rights. 2014; 14(26): $1-8$

[17] https://www.ncbi.nlm.nih.gov/books/NBK11737/. Accessed 28 December 2018.

[18] Musau S, Chee G, Patsika R, Malangalila E, Chitama D, Van Praag E, et al. Tanzania health system assessment 2010. Bethesda, MD: Health Systems. 2011.

[19] Vest JR, Gamm LD. Health information exchange: persistent challenges and new strategies. Journal of the American Medical Informatics Association. 2010; 17(3):288-94.

[20] Kripalani S, Jackson AT, Schnipper JL, Coleman EA. Promoting effective transitions of care at hospital discharge: a review of key issues for hospitalists. Journal of Hospital Medicine: an Official Publication of the Society of Hospital Medicine. 2007; 2(5):31423.

[21] https://iicd.org/documents/health-managementinformation-system-for-hospitals-lessons-learnedfrom-a-tanzanian-experience/. Accessed 28 December 2018.

[22] http://www.care2x.org/. Accessed 28 December 2018.

[23] https://www.healthdatacollaborative.org/fileadmin/upl oads/hdc/Documents/Country_documents/Tanzania_G OT-HOMIS_presentation_12Sept2017.pdf. Accessed 28 December 2018.

[24] https://docs.dhis2.org/2.22/en/user/html/dhis2_user_m anual_en.html. Accessed 28 December 2018.

[25] Lungo JH. The reliability and usability of district health information software: case studies from Tanzania. Tanzania Journal of Health Research. 2008; 10(1):39-45. 
[26] Valacich J, George J, Hoffer J. Essentials of systems analysis and design. Prentice Hall Press; 2014.

[27] Zhao J, Papapetrou P, Asker L, Boström H. Learning from heterogeneous temporal data in electronic health records. Journal of Biomedical Informatics. 2017; 65:105-19.

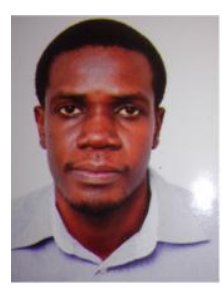

Geofrey Rweikiza is a student pursuing a Master's program in Information and Communications Sciences and Engineering at the Nelson Mandela African Institution of Science and Technology in Arusha, Tanzania. He holds a BSc. in Computer Science degree from St. Joseph University in Tanzania. He is also working as an ICT officer at the Tanzania Communications Regulatory Authority (TCRA). He is a Certified Network Security Systems Administrator (CNSSA). He is a Certified Secure Computer User (CSU) and Certified Oxygen Digital Forensic Expert. He is also a member of EC-COUNCIL.

Email: rweikizag@nm-aist.ac.tz

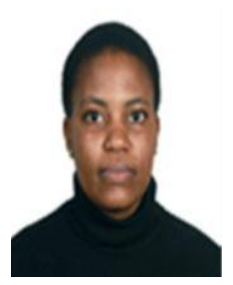

Dina Machuve is a Researcher and Lecturer at the Nelson Mandela African Institution of Science and Technology (NM-AIST), Tanzania. She holds a $\mathrm{PhD}$ in Information and Communication Science and Engineering in 2016, MS in Electrical Engineering obtained in 2008 and a BSc in Electrical Engineering. Her research interests are Data Science, Health and Agriculture Informatics. 